\title{
Retificação térmica, termorretificação, tratamento térmico, tratamento com calor ou modificação térmica?
}

\author{
Thermal treatment, heat treatment or thermal modification?
}

\author{
Djeison Cesar Batista ${ }^{\mathrm{I}}$
}

\begin{abstract}
Resumo
As pesquisas brasileiras sobre o processo que tem sido denominado de retificação térmica, termorretificação, tratamento térmico, tratamento com calor e modificação térmica vêm crescendo desde o ano 2000. Como a padronização de conceitos é algo importante para o desenvolvimento científico de uma determinada área, o objetivo deste trabalho foi realizar uma revisão bibliográfica sobre esse processo com a finalidade de se propor a nomenclatura técnica mais adequada para o mesmo em Língua Portuguesa, com fins de padronização. Foram revisados os artigos publicados no tema em 2015, em dez revistas científicas internacionais e em sete brasileiras. Também foi verificado um livro didático estrangeiro, o maior evento científico do tema e informações de empresas europeias detentoras de patentes de processo e que comercializam produtos. Com base análise de todas as fontes, para a padronização do processo e da madeira processada/produto originado, recomenda-se o uso das nomenclaturas técnicas modificação térmica e modificada termicamente, respectivamente. Tecnicamente, não está errado o uso dos termos tratamento térmico e tratada termicamente e seus equivalentes tratamento com calor e tratada com calor (apesar destas serem formas mais raras). Contudo, para não incorrer em erros de interpretação, essas nomenclaturas devem ser evitadas porque são genéricas e tradicionalmente empregadas para o consagrado processo de secagem artificial da madeira. Não se estimula o uso das nomenclaturas retificação térmica e termorretificação, bem como seus derivados retificada termicamente e termorretificada.
\end{abstract}

Palavras-chave: Madeira; Nomenclatura técnica; Processo de modificação térmica

\begin{abstract}
Brazilian studies of a process that has been variously called in Portuguese retificação térmica, termorretificação (both with no English correspondent), tratamento térmico (thermal treatment), tratamento com calor (heat treatment) or modificação térmica (thermal modification) have been increasing since 2000. Because the standardization of concepts is important for the scientific development of any subject, the aim of this article was to provide a literature review about this process, to propose the most appropriate technical nomenclature for it in Portuguese, aiming at standardization. We reviewed articles about this process, published in 2015, in ten international scientific journals and seven Brazilian journals. We also verified a foreign textbook, the largest scientific event on the subject and information on European companies that have process patents and products on the market were also verified. Based on all the verified sources, it is recommended the technical nomenclature 'modificação térmica' (thermal modification) for the process and 'modificada termicamente' (thermally modified) for the wood/products. Technically, it is not wrong to use the terms 'tratamento térmico' (thermal treatment) and 'tratada termicamente" (thermally treated) and their equivalents 'tratamento com calor (heat treatment) and 'tratada com calor' (heat treated). However, in order to avoid errors of interpretation, these nomenclatures should be avoided because they are generic and traditionally used for the well-established process of artificial drying of wood. It is also strongly discouraged the use of 'retificação térmica' and 'termorretificação' as well as their derivatives 'retificada termicamente' and 'termorretificada'.
\end{abstract}

Keywords: Wood; Technical nomenclature; Thermal modification process

\footnotetext{
I Engenheiro Florestal, Dr., Professor do Departamento de Ciências Florestais e da Madeira, Centro de Ciências Agrárias e Engenharias, Universidade Federal do Espírito Santo, Av. Gov. Carlos Lindenberg, 316, Centro, CEP 29550-000, Jerônimo Monteiro (ES), Brasil. djeison. batista@ufes.br (ORCID: 0000-0003-4427-2457)
} 


\section{Introdução}

Desde a década de 1920 vêm sendo conduzidos estudos sobre o processamento da madeira a elevadas temperaturas $\left(140^{\circ} \mathrm{C}-260^{\circ} \mathrm{C}\right)$, com diferentes equipamentos e meios de transmissão de calor (água, vapor de água, nitrogênio, óleo vegetal e metais derretidos), com o objetivo de alterar a composição química da madeira e melhorar algumas propriedades, tais como cor, estabilidade dimensional e resistência à biodeterioração. Para tanto, diferentes equipamentos e meios de transmissão de calor (água, vapor de água, nitrogênio, óleo vegetal e metais derretidos) tem sido utilizados. Esses estudos consolidaram-se na Europa a partir dos anos 1990, quando foram desenvolvidas as principais patentes que deram origem a processos em escala industrial, resultando em maior quantidade dessas indústrias e também no maior mercado consumidor desses produtos.

O desenvolvimento científico também ocorreu pela possibilidade de se agregar valor à madeira de pior qualidade, tais como aquelas de lenho juvenil, alburno e mesmo peças mescladas de cerne/alburno, pela homogeneização da cor. Como a madeira fica mais escura, em muitos casos, seus produtos têm sido apresentados como substitutos daqueles produzidos com madeira tropical.

Além do apelo ambiental pelo uso de madeira de reflorestamento e pelo não uso de espécies ameaçadas das florestas tropicais, o processo também traz a vantagem de aumentar a resistência à biodeterioração da madeira sem a impregnação ou utilização de nenhum produto químico biocida. Esses produtos têm sido utilizados tanto em ambientes externos quanto internos, conforme o processo e a matériaprima, tais como móveis, janelas, pisos e decks, componentes de fachadas, componentes de habitação (cladding), saunas, molduras, cercas, painéis colados lateralmente (edge glued pannels) e brinquedos de playground, ou seja, em produtos de maior valor agregado.

No Brasil, as pesquisas desse processo iniciaram na década de 1980, crescendo em frequência a partir do ano 2000. Portanto, trata-se um tema relativamente novo e pouco explorado pelos pesquisadores brasileiros, quando comparado com outros processos de industrialização da madeira, tais como desdobro, secagem, polpação, carbonização e produção de painéis. Não há padronização da nomenclatura do processo no Brasil, em que o mesmo tem sido designado como retificação térmica (BATISTA; KLITZKE, 2010; BORGES; QUIRINO, 2004;), termorretificação (CARVALHO et al., 2015; FONTOURA et al., 2015), tratamento térmico (PALERMO et al., 2015) ou modificação térmica (BATISTA; TOMASELLI; KLITZKE, 2011; POUBEL et al., 2015). Isso é inconveniente, porque a imperfeição de um conceito pode interferir negativamente no progresso de uma determinada área da ciência, pela dificuldade de comunicação entre seus pares (FERRARI, 1982).

$\mathrm{Na}$ argumentação da importância dos termos técnicos ou nomenclaturas que constituem um conceito, a conceituação é o elo fundamental da ciência, porque por este dispositivo trata-se de tornar inteligível os acontecimentos ou experiências que se dão no mundo real (FERRARI, 1982). A respeito de indústrias madeireiras, por exemplo, quando se usa a palavra "desdobro", os profissionais que atuam nessa área devem ter o mesmo entendimento: o processo em que toras são transformadas em madeira serrada pela ação de conjuntos máquina-ferramenta de corte. A secagem artificial da madeira, por exemplo, possui classificações bem distintas e consagradas das diferentes temperaturas utilizadas (baixas temperaturas, convencional e altas temperaturas), o que facilita a comunicação internacional tanto científica quanto industrial e comercial (SIMPSON, 1991).

O objetivo deste trabalho foi realizar uma revisão bibliográfica para verificar a nomenclatura técnica mais adequada em Língua Portuguesa que se ajusta ao seguinte conceito: processo que utiliza elevadas temperaturas $\left(140^{\circ} \mathrm{C}-260^{\circ} \mathrm{C}\right)$, com diferentes equipamentos e meios de transmissão de calor (água, vapor de água, nitrogênio e óleo vegetal), com o objetivo de causar alterações na composição química da madeira que irão resultar em melhoria de algumas propriedades (cor, estabilidade dimensional e resistência à biodeterioração). 


\section{Desenvolvimento}

\section{Nomenclatura técnica do processo na Europa}

Como a Europa é o principal continente no tocante ao processo que foi objeto desta revisão bibliográfica, seja em nível industrial ou científico, para se buscar uma padronização para a sua nomenclatura técnica foi necessário investigar as publicações daquele continente. Uma vez que a maioria das obras consultadas foi redigida em Língua Inglesa, apresentam-se as seis principais nomenclaturas técnicas analisadas e a forma como foram traduzidas para a Língua Portuguesa.

As três primeiras referem-se ao processo: i) thermal modification: modificação térmica; ii) thermal treatment: tratamento térmico; e iii) heat treatment: tratamento com calor. As três seguintes são derivadas das três primeiras e correspondentes, referem-se ao produto, ou seja, a madeira processada. Por isso foram apresentadas na forma feminina, para concordar em gênero com o substantivo feminino "madeira", já que em Língua Inglesa não há concordância em gênero para os adjetivos: i) thermally modified: modificada termicamente; ii) thermally treated: tratada termicamente; e iii) heat treated: tratada com calor.

Para a investigação sobre a nomenclatura técnica do processo na Europa foi importante considerar quatro referenciais bastante significativos do tema: um congresso, um livro-texto, as empresas detentoras das principais patentes e as publicações em revistas científicas.

\section{European Conference on Wood Modification}

Os congressos científicos (e seus similares) reúnem os principais pesquisadores de um determinado tema e região geográfica, portanto, são referenciais confiáveis para a busca de padronização de uma nomenclatura técnica. Isso se torna mais relevante à medida que um determinado congresso se consolide internacionalmente e possua um grande número de edições.

AECWM - European Conference on Wood Modification (Conferência Europeia Sobre Modificação da Madeira) teve a sua primeira edição no ano de 2003 em Ghent, na Bélgica (HUGHES; RAUTKARI; JONES, 2015), e em setembro de 2018 ocorreu a nona e última edição do evento, em Arnhem, Holanda (EUROPEAN CONFERENCE ON WOOD MODIFICATION, 2018). Ao longo de nove edições, a ECWM se consolidou como o mais importante evento científico mundial sobre as tecnologias de modificação da madeira, congregando as principais empresas e pesquisadores da área.

O programa tradicional da ECWM tem considerado aspectos industriais, econômicos, técnicos e científicos, além da questão ambiental, com foco principal nas modificações térmica (incluindo a óleotérmica), química e por impregnação, que possuem produção em escala industrial. Além dessas, também têm sido abordadas tecnologias emergentes, como os processos de superfície, que envolvem modificação química e biológica utilizando enzimas ou processos físicos, tais como ação mecânica e térmica, microondas e plasma (EUROPEAN CONFERENCE ON WOOD MODIFICATION, 2018).

\section{Wood modification: chemical, thermal and other processes}

Os livros são os principais materiais didáticos de consulta, principalmente para iniciantes em um determinado assunto. O material didático mais abrangente sobre modificação da madeira é o livrotexto Wood modification: chemical, thermal and other processes, de autoria de Hill (2006), que à época da publicação era professor sênior da School of Agricultural and Forest Sciences, Universidade do País de Gales. Na obra, Hill (2006) classificou em quatro os métodos de modificação da madeira: química, térmica, de superfície e de impregnação.

A produção de material didático se intensifica à medida que um determinado tema se torna uma disciplina em cursos formais, tais como os de graduação e pós-graduação. Como a modificação da madeira é um tema relativamente novo, espera-se que mais material didático seja produzido futuramente.

Além do livro de Hill (2006), mencionam-se duas obras sobre modificação térmica da madeira: ThermoWood ${ }^{\mathbb{R}}$ Handbook (INTERNATIONAL THERMOWOOD ASSOCIATION, 2003) e Processes and 
properties of thermally modified wood manufactured in Europe (MILITZ; ALTGEN, 2014). A primeira obra é um manual sobre o processo industrial finlandês ThermoWood ${ }^{\circledR}$; a segunda, mais abrangente, é um capítulo de livro no qual consta uma revisão bibliográfica sobre os principais processos europeus de modificação térmica da madeira.

A modificação térmica da madeira é um processo ativo, e é definido como a aplicação de calor à madeira com o objetivo de causar melhorias no desempenho do material. É classificado como ativo porque o calor causa modificações na natureza química da madeira, que resultam em mudanças nas suas propriedades (HILL, 2006). Com base na ECWM e nas obras de Hill (2006) e Militz e Altgen (2014), modificação térmica é a nomenclatura técnica recomendada para o processo objeto desta revisão, e que deveria ser utilizada para padronização em nível mundial. A madeira e os produtos derivados seriam denominados modificada termicamente e modificados termicamente, respectivamente. Contudo, ainda é necessário fazer a análise das patentes europeias e as publicações nas revistas científicas.

\section{Patentes europeias de processos}

As revisões bibliográficas mais recentes do tema também abordaram as patentes europeias em nível industrial (GÉRARDIN, 2016; MILITZ; ALTGEN, 2014), em que sete foram identificadas: ThermoWood, Platowood, Retification, Oil Heat Treatment, Vacu ${ }^{3}$, Termo Vuoto e processo a alta pressão. Dessa forma, foram selecionadas informações oficiais das empresas detentoras para verificar como as mesmas designam tecnicamente o processo.

\section{ThermoWood $^{\mathbb{B}}$}

Na Finlândia encontra-se o grupo mais conhecido mundialmente que produz e comercializa os produtos desse processo: a International ThermoWood Association (Associação Internacional ThermoWood), anteriormente denominada Finnish ThermoWood Association (Associação Finlandesa ThermoWood). Isso se deve a alguns fatores: organização institucional, investimento em pesquisa e desenvolvimento (equipamentos, processo e produtos), normatização, padronização, certificação e divulgação da marca e dos produtos.

Os produtos comercializados com a marca registrada ThermoWood ${ }^{\circledR}$ adquiriram tanta popularidade em nível internacional que, pela falta de padronização da nomenclatura técnica, ThermoWood ${ }^{\circledR}$ passou a ser sinônimo tanto do processo quando dos produtos. Isso levou, inclusive, a disputas judiciais dos proprietários da marca com outras empresas que faziam uso indevido da mesma (INTERNATIONAL THERMOWOOD ASSOCIATION, 2018).

Soma-se à estratégia de divulgação a publicação da obra mais conhecida dessa Associação, o ThermoWood ${ }^{\mathbb{B}}$ Handbook (INTERNATIONAL THERMOWOOD ASSOCIATION, 2003), mencionada na seção anterior. Trata-se de um manual didático sobre o processo e os produtos comercializados com a marca ThermoWood ${ }^{\circledR}$. Esse é um dos primeiros materiais didáticos do tema em que foi dado enfoque científico, e não apenas comercial.

Nessa obra é informado que se trata de um processo em escala industrial de tratamento com calor (industrial scale heat-treatment process). Por outro lado, a página inicial do sítio eletrônico oficial da International ThermoWood Association menciona que madeira modificada termicamente (thermally modified wood) é utilizada nos seus produtos (INTERNATIONAL THERMOWOOD ASSOCIATION, 2018).

\section{Platowood $^{\mathbb{R}}$}

A empresa holandesa Platowood produz e comercializa principalmente molduras, painéis colados lateralmente (uso interno e externo) e janelas com o nome comercial idêntico ao da empresa (PLATOWOOD, 2018a). Em um de seus documentos técnicos, o processo é descrito em detalhes e classificado como modificação hidrotérmica (hydrothermal modification) (PLATOWOOD, 2018b), que é uma variação da modificação térmica. Entretanto, o processo descrito pela empresa se caracteriza como 
higrotérmico (hygrothermal) em vez de hidrotérmico (hydrothermal), porque utiliza vapor saturado em um ambiente a alta pressão, ao passo que no hidrotérmico a madeira deve ser imersa em água (HILL, 2006).

\section{Retification $^{\circledR}$}

O processo Retification foi desenvolvido na École Nationale Supérieure des Mines de SaintÉtienne (EMSE), França, em parceria com a empresa proprietária da patente New Option Wood (NOW) e a empresa Rey, fabricante do equipamento. No sítio eletrônico oficial da EMSE podem ser encontradas informações do processo, dentre as quais a que designa o mesmo como um tratamento térmico da madeira (GUYONNET, 2007).

Foi realizada uma busca na rede mundial de computadores (Internet), utilizando-se as palavraschave retification e new option wood, porém, não foram encontrados sítios eletrônicos oficiais de empresas detentoras de permissão do uso da patente. Dessa forma, não foi possível verificar alguma possível atualização da nomenclatura técnica do processo.

\section{Oil Heat Treatment (OHT)}

O processo oil heat treatment $(\mathrm{OHT})$ é realizado pela imersão da madeira em óleo vegetal aquecido, totalmente diferente dos demais, nos quais a madeira é aquecida por vapor de água ou gás nitrogênio. As empresas Menz Holz (alemã) e Oléoboís (francesa) são as que mais produzem madeira usando o OHT, em que a primeira denomina o processo como Menz OHT (GÉRARDIN, 2016).

De acordo com a tradução do próprio nome, o processo é apresentado como um tratamento com calor, e a empresa francesa adota esta nomenclatura (OLÉOBOÍS, 2018). Contudo, conforme um catálogo de 2012 da empresa Menz Holz, a mesma designa o processo como tratamento térmico (MENZ HOLZ, 2012 citado por BATISTA, 2012). Em busca realizada na Internet, não foi encontrado um sítio eletrônico oficial da empresa Menz Holz para verificar alguma possível atualização da nomenclatura técnica.

\section{Condições pressurizadas}

Esta classificação refere-se a um processo hidrotérmico comercializado pelas empresas dinamarquesas IWT/Moldrup e WTT (Wood Treatment Technology) e pela empresa holandesa FirmoLin Technologies, que consiste na aplicação de vapor de água na madeira em um ambiente de alta pressão, utilizando-se um equipamento semelhante a uma autoclave de usinas de preservação de madeira (GÉRARDIN, 2016; MILITZ; ALTGEN, 2014).

A empresa IWT/Moldrup denomina o processo Moldrup-SSP ${ }^{\circledR}$ de tratamento térmico (IWT MOLDRUP, 2018), ao passo que para a empresa WTT o processo WTT Thermo Treat $2.0^{\circledR}$ é denominado thermo treatment (sem tradução) e tratamento com calor; e os produtos como thermo treated (sem tradução) e madeira modificada termicamente (WOOD TREATMENT TECHNOLOGY, 2018). A empresa FirmoLin Technologies denomina o processo FirmoLin ${ }^{\circledR}$ modificação térmica (FIRMOLIN, 2018). Apesar de esta parte da revisão se referir às patentes europeias, destaca-se que o processo brasileiro de modificação térmica (TanWood antigo VAP HolzSysteme), de propriedade da empresa TWBrazil, também se enquadra na classificação de condições pressurizadas (BATISTA, 2012).

\section{Condições com vácuo}

Esta classificação refere-se aos processos que utilizam, além de elevadas pressões, aplicação de vácuo como forma de minimizar a concentração de oxigênio no ambiente (GÉRARDIN, 2016). Duas empresas europeias possuem patentes que se enquadram nesta classificação e o equipamento usado por ambas se assemelha a uma câmara de secagem a vácuo, com as devidas adaptações e modificações.

A empresa alemã Timura utiliza vacuum press dewatering method ou Vacu ${ }^{3}$ para denominar o processo (MILITZ; ALTGEN, 2014), em que a madeira é empilhada em camadas intercaladas com placas térmicas, que recebem um carregamento adicional de 70 toneladas, o que aumenta o contato entre o 
conjunto, minimiza defeitos e facilita a transferência de calor por condução. A empresa denomina o processo de tratamento térmico e os produtos por uma grande diversidade de nomes: thermal wood e thermo wood (ambos sem tradução), madeira modificada termicamente, madeira processada termicamente, madeira tratada termicamente e madeira tratada com calor (TIMURA, 2018). A empresa italiana Wood Drying Engineering Maspell (WDE Maspell) possui um pedido de patente chamado Termo Vuoto ${ }^{\circledR}$, e denomina o processo como tratamento a altas temperaturas, sem apresentar qualquer nomenclatura específica para os produtos (WDE MASPELL, 2018).

Analisando-se as informações das empresas detentoras das patentes, verificou-se grande inconsistência, indicando que essa análise não foi esclarecedora para atender ao objetivo proposto na revisão. A maioria das empresas somente possui informações veiculadas em sítios eletrônicos, em que não se verificou compromisso com a exatidão da nomenclatura técnica do processo, mas basicamente, enfoque comercial, o que era esperado.

As informações mais organizadas sobre processo e produtos, veiculadas oficialmente pelas empresas e com algum enfoque técnico e científico, foram encontradas para o ThermoWood (INTERNATIONAL THERMOWOOD ASSOCIATION, 2003; 2018) e o Platowood (PLATOWOOD 2018a; 2018b). Para maiores informações sobre os processos industriais europeus são recomendados, além da busca nos sítios eletrônicos das empresas, os seguintes trabalhos de revisão: Rapp (2001), Xie, Liu e Sun (2002), Homan e Jorissen (2004), Esteves e Pereira (2009), Militz e Altgen (2014), Gérardin (2016) e Sandberg e Kutnar (2016).

\section{Revistas científicas internacionais}

Conforme experiência com o tema, foram selecionadas dez revistas científicas internacionais que publicam artigos sobre o processo objeto desta revisão bibliográfica, que foram consultadas via sítio eletrônico oficial na Internet, restringindo-se inicialmente aos artigos publicados em 2015. A consulta foi realizada no período entre 18 e 22 de janeiro de 2016 e verificou-se que algumas revistas já possuíam publicações do ano de 2016, que também foram consultadas.

O Qualis das revistas foi consultado na área de avaliação de Ciências Agrárias I, e se refere ao quadriênio 2013-2016; exceto para Biodegradation, que possuía Qualis somente para o triênio 2010-2012". $\mathrm{Na}$ Tabela 1 encontram-se as informações das dez revistas analisadas, ordenadas de forma decrescente de acordo com a quantidade de artigos encontrados.

Tabela 1 - Informações das revistas científicas internacionais analisadas.

Table 1 - Information from international scientific journals analyzed.

\begin{tabular}{|c|c|c|c|}
\hline Revista & ISSN* & Endereço eletrônico de consulta & Qualis** \\
\hline BioResources & $1930-2126$ & https://www.ncsu.edu/BioResources/ & $\mathrm{A} 2$ \\
\hline Holzforschung & 0018-3830 & http://www.degruyter.com/view/j/hfsg & $\mathrm{A} 2$ \\
\hline Maderas: Ciencia y Tecnología & $0718-221 X$ & $\begin{array}{l}\text { http://www.scielo.cl/scielo.php?script=sci_ } \\
\text { serial\&pid=0718-221X\&lng=es\&nrm=iso }\end{array}$ & B1 \\
\hline $\begin{array}{l}\text { European Journal of Wood and Wood } \\
\text { Products }\end{array}$ & $1436-736 \mathrm{X}$ & http://link.springer.com/journal/107 & B1 \\
\hline Journal of Materials Science & $1573-4803$ & http://link.springer.com/journal/10853 & $\mathrm{A} 2$ \\
\hline Annals of Forest Science & $1286-4560$ & http://link.springer.com/journal/13595 & A1 \\
\hline Wood Science and Technology & 0043-7719 & http://link.springer.com/journal/226 & $\mathrm{A} 2$ \\
\hline Biodegradation & 0923-9820 & http://link.springer.com/journal/10532 & $\mathrm{A} 2$ \\
\hline Bioresource Technology & $0960-8524$ & $\begin{array}{l}\text { http://www.journals.elsevier.com/bioresource- } \\
\text { technology/ }\end{array}$ & A1 \\
\hline $\begin{array}{l}\text { International Biodeterioration and } \\
\text { Biodegradation }\end{array}$ & 0964-8305 & $\begin{array}{c}\text { http://www.journals.elsevier.com/international- } \\
\text { biodeterioration-and-biodegradation/ }\end{array}$ & B1 \\
\hline
\end{tabular}


Foram encontrados 50 artigos sobre o tema, publicados em sete das dez revistas avaliadas. Embora tenham publicado artigos do tema no passado, o mesmo não se verificou no período analisado para as revistas Biodegradation, Bioresource Technology e International Biodeterioration and Biodegradation. Depois de coletados, foram buscadas principalmente as seguintes palavras-chave nos artigos: thermal modification, thermal treatment e heat treatment, para qualificar o processo, e thermally modified, thermally treated e heat treated, para qualificar o material/produto.

Dos artigos da revista Holzforschung foram analisados apenas o título, o resumo e as palavraschave, porque a editora não disponibilizava os trabalhos completos para consulta online de forma gratuita. A única exceção desta revista foi artigo de Sonderegger et al. (2015) que estava disponível na íntegra para download.

Apesar de não ser europeia, a maior quantidade de artigos (13) foi encontrada na revista BioResources, editada pela Universidade da Carolina do Norte, Estados Unidos da América (ČERMÁK et al., 2015; CIRULE et al., 2016; FAJDIGA et al., 2016; GAFF et al., 2015; GAŠPARÍK et al., 2015; GAŠPARÍK; GAFF, 2015; KVIETKOVÁ, 2015; KVIETKOVÁ et al., 2015; LING et al., 2016; TORNIAINEN; ELUSTONDO; DAGBRO, 2016; YANG; CHENG; HAN, 2015; YANG et al., 2015; ZHANG et al., 2015).

A revista Holzforschung ficou na segunda posição, com 11 artigos publicados (BIZIKS et al., 2015; HUGHES; HILL; PFRIEM, 2015; JAVED et al., 2015; KIM et al., 2015a; 2015b; LI et al., 2015; PEARSON; ORMARSSON; GABBITAS, 2015; SONDEREGGER et al., 2015; TRCALA; ČERMÁK, 2015; WANG et al., 2015; WILLEMS et al., 2015).

A revista chilena Maderas: Ciencia y Tecnología, editada pela Universidade de Bío-Bío, ficou empatada com a Holforschung, com 11 artigos publicados (BAL, 2015; BATISTA et al., 2015; CANDELIER et al., 2015; ELAIEB et al., 2015; FABIYI; OGUNLEYE, 2015; HERMOSO et al., 2015; KORKUT; AYTIN, 2015; SAEI; MOHEBBY; ABDEH, 2015; SIVRIKAYA et al., 2015; YALCIN; SAHIN, 2015; ZANUNCIO et al., 2015).

A revista European Journal of Wood and Wood Products ficou na quarta posição, com nove artigos publicados (ALTGEN; MILITZ, 2015; BASTANI; ADAMOPOULOS; MILITZ, 2015a; 2015b; KUZMAN et al., 2015; PANDEY; KUMAR; SRINIVAS, 2015; ZIGON et al., 2015; SALMAN et al., 2016; ZAUER et al., 2016; ZHAN; AVRAMIDIS, 2016).

Foram encontrados dois artigos em cada uma das seguintes revistas, que foram analisados conjuntamente: Annals of Forest Science, Journal of Materials Science e Wood Science and Technology (ČERMÁK et al., 2016; GÉRARDIN, 2016; HAMADA et al., 2016; RINGMAN et al., 2015; TODOROVIĆ; POPOVIĆ; MILIĆ, 2015; TUKIAINEN; HUGHES, 2016).

$\mathrm{Na}$ Tabela 2 encontra-se o resumo das frequências das nomenclaturas técnicas encontradas nos 50 artigos, agrupados por revista. As frequências foram calculadas dividindo-se o número de artigos em que uma determinada nomenclatura foi apresentada, pelo número total de artigos da respectiva revista, multiplicado por 100. Independentemente de uma nomenclatura ter sido predominante ou não em um artigo, caso tenha sido apresentada pelo menos uma vez, a mesma foi contada para o cálculo da frequência. Essa decisão facilitou sobremaneira a organização dos resultados.

Antes da discussão dos resultados da Tabela 2, três artigos merecem destaque. O artigo de Li et al. (2015), publicado na Holzforschung, foi o único no qual a madeira não foi o material estudado, mas sim o bambu, em que na porção disponível para acesso não foi mencionada a espécie. Apenas um artigo foi publicado em Língua Espanhola (Maderas: Ciencia y Tecnología), com resumo e palavraschave em Língua Inglesa, no qual foi encontrada apenas a designação thermally modified (modificada termicamente) (HERMOSO et al., 2015). Foram encontrados os seguintes termos em Língua Espanhola, com seus respectivos correspondentes em Língua Portuguesa: modificada térmicamente (modificada termicamente), modificación térmica (modificação térmica) e tratamiento térmico (tratamento térmico). Em apenas um artigo (European Journal of Wood and Wood Products) foi adotada a nomenclatura modificada com calor (heat-modified) para designar a madeira/produto (KUZMAN et al., 2015). 
Tabela 2 - Resumo das frequências das nomenclaturas técnicas.

Table 2 - Summary of technical nomenclature frequencies.

\begin{tabular}{|c|c|c|c|c|c|c|}
\hline \multirow[b]{3}{*}{ Revista } & \multicolumn{6}{|c|}{ Frequência da nomenclatura técnica (\%) } \\
\hline & \multicolumn{3}{|c|}{ Processo } & \multicolumn{3}{|c|}{ Produto/Madeira } \\
\hline & $\begin{array}{c}\text { Modificação } \\
\text { térmica }\end{array}$ & $\begin{array}{c}\text { Tratamento } \\
\text { com calor }\end{array}$ & $\begin{array}{c}\text { Tratamento } \\
\text { térmico }\end{array}$ & $\begin{array}{l}\text { Modificada } \\
\text { termicamente }\end{array}$ & $\begin{array}{c}\text { Tratada } \\
\text { com } \\
\text { calor }\end{array}$ & $\begin{array}{c}\text { Tratada } \\
\text { termicamente }\end{array}$ \\
\hline BioResources & 69 & 54 & 62 & 77 & 23 & 46 \\
\hline Holzforschung & 64 & 45 & 36 & 64 & 0 & 18 \\
\hline $\begin{array}{l}\text { Maderas: Ciencia y } \\
\text { Tecnología }\end{array}$ & 73 & 82 & 82 & 73 & 73 & 27 \\
\hline $\begin{array}{l}\text { European Journal } \\
\text { of Wood and Wood } \\
\text { Products }\end{array}$ & 78 & 89 & 44 & 56 & 78 & 33 \\
\hline Três revistas* & 100 & 66 & 83 & 100 & 50 & 0 \\
\hline Média & 77 & 67 & 61 & 74 & 45 & 25 \\
\hline
\end{tabular}

*Annals of Forest Sciences, Journal of Materials Science e Wood Science and Technology.

Antes da discussão dos resultados da Tabela 2, três artigos merecem destaque. $\mathrm{O}$ artigo de Li et al. (2015), publicado na Holzforschung, foi o único no qual a madeira não foi o material estudado, mas sim o bambu, em que na porção disponível para acesso não foi mencionada a espécie. Apenas um artigo foi publicado em Língua Espanhola (Maderas: Ciencia y Tecnología), com resumo e palavras-chave em Língua Inglesa, no qual foi encontrada apenas a designação thermally modified (modificada termicamente) (HERMOSO et al., 2015). Foram encontrados os seguintes termos em Língua Espanhola, com seus respectivos correspondentes em Língua Portuguesa: modificada térmicamente (modificada termicamente), modificación térmica (modificação térmica) e tratamiento térmico (tratamento térmico). Em apenas um artigo (European Journal of Wood and Wood Products) foi adotada a nomenclatura modificada com calor (heat-modified) para designar a madeira/produto (KUZMAN et al., 2015).

Em média, houve preferência do uso dos termos técnicos modificação térmica (77\%) e madeira modificada termicamente (74\%), para o processo e produto, respectivamente; ao passo que houve menor preferência pelo uso de tratamento térmico $(61 \%)$ e madeira tratada termicamente $(25 \%)$. Esse resultado está em acordo com as obras de Hill (2006) e Militz e Altgen (2014), e a ECWM, apresentados anteriormente.

Contudo, como era esperado, não houve unanimidade na utilização das nomenclaturas técnicas, quer seja em uma mesma revista ou entre revistas. A análise mostrou-se mais complexa do que o esperado, porque é muito comum em um mesmo artigo os autores utilizarem mais de uma nomenclatura para o processo ou o produto, embora haja sempre uma nomenclatura predominante.

Isso pode ser observado pela soma das frequências por revista, separadamente para processo e produto, que sempre ultrapassam $100 \%$. A única exceção foi a Holzforschung, na qual a soma das frequências para o produto atingiu apenas $82 \%$, uma vez que o texto avaliado se restringiu apenas ao título, resumo e palavras-chave, conforme explicado anteriormente. Em três artigos dessa revista (BIZIKS et al., 2015; PEARSON; ORMARSSON; GABBITAS, 2015; TRCALA; ČERMÁK, 2015) não foi possível identificar como os autores denominavam o produto.

Em apenas sete dos 50 artigos avaliados (14\%) os autores foram absolutamente coerentes, utilizando em todo o texto apenas uma nomenclatura para o processo e para o produto, que podem ser separados em dois grupos: modificação térmica e modificada termicamente, com quatro artigos (ALTGEN; MILITZ, 2015; BATISTA et al., 2015; GAŠPARÍK et al., 2015; TORNIAINEN; ELUSTONDO; DAGBRO, 2016); e tratamento com calor e tratada com calor, com três artigos (BASTANI; ADAMOPOULOS; MILITZ, 2015a; KUZMAN et al., 2015; YANG et al., 2015). Os artigos 
da revista Holzforschung não foram incluídos nessa análise pelos motivos anteriormente mencionados. Revistas científicas brasileiras

Neste ponto da revisão foi possível perceber a preferência da literatura europeia pelas nomenclaturas técnicas modificação térmica e modificada termicamente, para processo e madeira/ produto, respectivamente. Entretanto, para atender ao problema de pesquisa levantado para esta revisão, ainda foi necessário verificar as publicações científicas do tema em revistas brasileiras.

Conforme experiência, foram selecionadas sete revistas científicas brasileiras que publicam artigos sobre o processo objeto desta revisão bibliográfica, que foram consultadas no dia 28 de janeiro de 2016 via sítio eletrônico oficial na Internet, restringindo-se apenas aos artigos publicados em 2015. O Qualis foi consultado na área de avaliação de Ciências Agrárias I, e refere-se ao ano de 2014 e na Tabela 3 encontram-se as informações das sete revistas analisadas, por ordem alfabética.

\section{Tabela 3 - Informações das revistas científicas brasileiras analisadas.}

Table 3 - Information from the assessed Brazilian scientific journals.

\begin{tabular}{|c|c|c|c|}
\hline Revista & ISSN* & Endereço eletrônico de consulta & Qualis** \\
\hline $\begin{array}{l}\text { Revista } \\
\text { Árvore }\end{array}$ & $1806-9088$ & $\begin{array}{c}\mathrm{http}: / / \text { www.scielo.br/scielo.php?script }=\text { sci_serial\&pid }=0100- \\
6762 \& \operatorname{lng}=\text { en\&nrm=iso }\end{array}$ & B1 \\
\hline Cerne & $0104-7760$ & $\begin{array}{c}\text { http://www.scielo.br/scielo.php?script }=\text { sci_serial\&pid }=0104- \\
7760 \& \operatorname{lng}=\text { en\&nrm=iso }\end{array}$ & $\mathrm{B} 1$ \\
\hline $\begin{array}{l}\text { Ciência } \\
\text { Florestal }\end{array}$ & 0103-9954 & $\begin{array}{c}\text { http://cascavel.ufsm.br/revistas/ojs-2.2.2/index.php/ } \\
\text { cienciaflorestal/index }\end{array}$ & $\mathrm{B} 1$ \\
\hline $\begin{array}{l}\text { Ciência da } \\
\text { Madeira }\end{array}$ & $2177-6830$ & $\begin{array}{c}\text { https://periodicos.ufpel.edu.br/ojs2/index.php/ } \\
\text { cienciadamadeira }\end{array}$ & B3 \\
\hline Floresta & $1982-4688$ & http://ojs.c3sl.ufpr.br/ojs2/index.php/floresta & B1 \\
\hline $\begin{array}{l}\text { Floresta e } \\
\text { Ambiente }\end{array}$ & $1415-0980$ & http://www.floram.org/ & $\mathrm{B} 1$ \\
\hline $\begin{array}{l}\text { Scientia } \\
\text { Forestalis }\end{array}$ & $1413-9324$ & http://www.ipef.br/publicacoes/scientia/edicoes.asp & B1 \\
\hline
\end{tabular}

*International Standard Serial Number. **Consulta realizada em 30 de janeiro de 2018, no sítio eletrônico https://sucupira.capes.gov.br/sucupira/ public/consultas/coleta/veiculoPublicacaoQualis/listaConsultaGeralPeriodicos.jsf.

Foram encontrados apenas quatro artigos publicados no tema, todos em Língua Portuguesa, com resumo e palavras-chave em Língua Inglesa: dois na revista Ciência da Madeira (CARVALHO et al., 2015; FONTOURA et al., 2015), um na revista Scientia Forestalis (POUBEL et al., 2015) e um na revista Ciência Florestal (PALERMO et al., 2015). Depois de coletados, foram buscadas principalmente as seguintes palavras-chave nos artigos: retificação térmica, termorretificação, modificação térmica, tratamento térmico e tratamento com calor, para qualificar o processo; e retificada termicamente, termorretificada, modificada termicamente, tratada termicamente e tratada com calor, para qualificar a madeira/produto.

As traduções mencionadas na discussão a seguir referem-se ao abstract dos artigos, escritos em Língua Inglesa. Carvalho et al. (2015) utilizaram predominantemente os termos termorretificação e termorretificada para designar, respectivamente, o processo e o produto. Esses termos foram traduzidos para a Língua Inglesa como heat treatment e heat treated, respectivamente. Com menor frequência os autores também empregaram tratamento térmico para o processo, porém, com a mesma tradução para a Língua Inglesa de termorretificação: heat treatment. De fato, conforme será discutido posteriormente, não há tradução para a Língua Inglesa dos vocábulos termorretificação e termorretificada.

Fontoura et al. (2015) utilizaram predominantemente termorretificação para o processo, com a tradução heat treatment, e com menor frequência tratamento térmico, com a tradução thermal treatment. Os autores não deram muito enfoque ao produto, mas quando o mencionaram empregaram tratada termicamente, com a tradução heat treated. Palermo et al. (2015) empregaram somente os termos 
tratamento térmico e tratada termicamente para se referirem, respectivamente, ao processo e à madeira/ produto, os traduzindo como heat treatment e heat treated.

Por fim, Poubel et al. (2015) utilizaram predominantemente para o processo a nomenclatura modificação térmica, com a tradução thermal modification, e com menor frequência tratamento térmico, com a tradução heat treatment. Os autores também não deram muito enfoque ao produto, mas quando o mencionaram, o denominaram modificada termicamente, com a tradução heat treated.

Nas revistas científicas internacionais foram identificados predominantemente seis nomenclaturas técnicas, três para o processo e três para a madeira/produto e o mesmo ocorreu na literatura brasileira, porém, com um diferencial. No Brasil, os autores nomearam o processo como tratamento térmico, modificação térmica e termorretificação; ao passo que a madeira/produto foi nomeada como tratada termicamente, modificada termicamente e termorretificada.

As nomenclaturas internacionais não utilizadas pelos autores brasileiros foram tratamento com calor e sua correspondente tratada com calor e, em seus lugares, foram apresentados os termos termorretificação e termorretificadas. Assim, observa-se que estes dois últimos não possuem correspondente em Língua Inglesa, sendo nomenclaturas usadas tipicamente pelos pesquisadores brasileiros.

Apesar de os termos tratamento com calor/tratada com calor não serem utilizados no Brasil, suas traduções heat treatment/heat treated foram empregadas por todos os autores brasileiros. Para reforçar que termorretificação e termorretificada são termos tipicamente brasileiros, eles foram traduzidos como heat treatment/heat treated, por não haver equivalentes em Língua Inglesa.

Embora este não tenha sido objetivo da revisão, notou-se que as traduções dos termos para a Língua Inglesa não foram fiéis: tratamento térmico (thermal treatment) foi traduzido como heat treatment (tratamento com calor); tratada termicamente (thermally treated) e modificada termicamente (thermally modified) foram traduzidas como heat treated (tratada com calor).

Dos 50 artigos encontrados em revistas internacionais, apenas dois, publicados em Língua Inglesa na revista Maderas: Ciencia y Tecnología, são de autores brasileiros. Batista et al. (2015) utilizaram predominantemente as nomenclaturas modificação térmica e modificada termicamente (thermal modification e thermally modified) e, da mesma forma, Zanuncio et al. (2015) empregaram tratamento com calor e tratada com calor (heat treatment e heat treated).

Da mesma forma que não há padronização na literatura internacional, o mesmo ocorre entre os autores brasileiros que publicaram nas revistas brasileiras e estrangeiras, o que reforça a hipótese inicial desta revisão bibliográfica, em se propor uma padronização das nomenclaturas técnicas do processo em análise.

Modificação térmica e modificada termicamente

Tendo como base os quatro referenciais adotados, verificou-se que em pelo menos três (HILL, 2006; EUROPEAN CONFERENCE ON WOOD MODIFICATION, 2018 e publicações internacionais) existe a preferência pelo uso das nomenclaturas técnicas modificação térmica e modificada termicamente. Considerando-se os artigos publicados por autores brasileiros em 2015, independentemente do local de publicação da revista, verificou-se em dois a mesma forma de utilização (BATISTA et al., 2015; POUBEL et al., 2015).

Conforme a revisão bibliográfica, os primeiros pesquisadores a utilizarem essas nomenclaturas em um artigo científico foram Tjeerdsma et al. (1998), que publicaram seu trabalho na antiga revista Holz als Roh-und Werkstoff (atual European Journal of Wood and Wood Products). Da mesma forma, os primeiros pesquisadores brasileiros a empregarem essas nomenclaturas em revistas internacional e brasileira, respectivamente, foram Calonego, Severo e Furtado (2010) - Bioresource Technology - e Batista, Tomaselli e Klitzke (2011) - Ciência Florestal.

Antes de se sugerir a adoção dessas nomenclaturas técnicas, é importante discutir porque as demais não devem ser utilizadas. Foi dado enfoque apenas à nomenclatura do processo e não do produto, haja vista que esta é derivada daquela. 
Tratamento térmico e tratamento com calor

De acordo com a discussão da seção que tratou das patentes dos processos europeus, a modificação térmica é um processo de industrialização da madeira, assim como a preservação, secagem, desdobro e polpação, por exemplo. Obviamente, todos esses processos industriais podem ser realizados em menor escala, em laboratório, e sujeitos à investigação científica.

Para esta análise, o significado mais pertinente para o substantivo masculino tratamento vem da Medicina: "procedimento médico que visa curar, aliviar ou prevenir" (ANJOS; FERREIRA, 2000). A Estatística o tem empregado, porém de forma ampliada para qualquer área do conhecimento, sendo utilizado em delineamentos experimentais. Conforme Pimentel-Gomes e Garcia (2002), para os delineamentos experimentais estatísticos, tratamento significa os métodos de estudo que são aplicados às unidades experimentais ou parcelas.

Por exemplo, um pesquisador ao coletar $4 \mathrm{~m}^{3}$ de madeira serrada separa ao acaso quatro lotes de 1 $\mathrm{m}^{3}$, chamados parcelas. A madeira da parcela 1 permanece em sua forma original (controle ou testemunha) e a madeira das parcelas 2 a 4 é submetida, respectivamente, às temperaturas de $140{ }^{\circ} \mathrm{C}, 160{ }^{\circ} \mathrm{C}$ e $180{ }^{\circ} \mathrm{C}$ por 45 minutos. Por fim, têm-se quatro tratamentos, porque quatro métodos de estudo diferentes foram aplicados aos lotes de madeira serrada.

Como no processo de modificação térmica são utilizadas altas temperaturas (maiores que 140 ${ }^{\circ} \mathrm{C}$ ), durante o delineamento experimental tem sido comum os pesquisadores agruparem seus métodos de estudo na nomenclatura tratamento térmico em oposição à madeira-controle, testemunha ou não tratada. $\mathrm{O}$ adjetivo térmico (ou sua forma feminina térmica) significa relativo ao calor ou que o conserva (ANJOS; FERREIRA, 2000) e, dessa forma, as designações tratamento térmico e tratamento com calor seriam sinônimas. Embora tenha sido apresentada uma definição semântica, do ponto de vista da Termodinâmica, nunca se considera que o calor esteja armazenado em um corpo, mas como a energia em trânsito de um corpo para outro quando ocorre diferença de temperatura (SMITH; VAN; ABBOTT, 2011).

Assim, conclui-se que vários tipos de tratamentos empregados na madeira e que usam calor, tais como o congelamento, aquecimento e, mais comumente a secagem, podem ser genericamente considerados "térmicos". Portanto, para se evitar qualquer erro de interpretação, as nomenclaturas tratamento térmico e tratamento com calor, por serem muito genéricas e abrangentes, não devem ser utilizadas para se referir ao processo industrial em análise nesta revisão.

O artigo de Takeshita e Jankowsky (2015), por exemplo, menciona a nomenclatura tratamento térmico no seu título. Ao se analisar a metodologia, compreende-se que a madeira, após passar pelo processo de secagem, foi submetida a 12 diferentes tratamentos resultantes da combinação de três temperaturas e quatro tempos. Contudo, as temperaturas empregadas $\left(60^{\circ} \mathrm{C}, 75^{\circ} \mathrm{C}\right.$ e $\left.90^{\circ} \mathrm{C}\right)$ não são capazes de causar modificação na composição química da parede celular da madeira. Este não era o objetivo dos autores e, conforme apresentado anteriormente, os métodos aplicados por eles estão corretamente designados como tratamentos térmicos.

A norma $\mathrm{n}^{\circ} 15$ das Normas Internacionales para Medidas Fitosanitarias - NIMF $\mathrm{N}^{\circ} 15$ (FOOD AND AGRICULTURE ORGANIZATION OF UNITED NATIONS, 2003) diz respeito às diretrizes da utilização de madeira para embalagens utilizadas em exportações. Nessa norma, que é de utilização mundial, apresenta-se o conceito de tratamento térmico, que é o processo em que a madeira é aquecida de forma a se atingir $56{ }^{\circ} \mathrm{C}$ na metade da espessura, por pelo menos 30 minutos. A madeira nessas condições estaria adequada para ser utilizada como embalagem de produtos exportados, recebendo um selo reconhecido internacionalmente com as iniciais HT (heat treated).

É importante mencionar uma publicação do programa Value to Wood, coordenado pela associação canadense sem fins lucrativos FPInnovations (2003), intitulada Thermally Modified Wood (madeira modificada termicamente). Um dos objetivos dessa publicação, já àquela época, era diferenciar os processos de modificação térmica e tratamento térmico. Conforme o documento, tratamento térmico (HT-KD) corresponde à secagem em estufa (kiln drying - KD) para atender aos requisitos internacionais de madeira usada em embalagens (NIMF-15), ao passo que modificação

Ci. Fl., Santa Maria, v. 29, n. 1, p. 463-480, jan./mar., 2019 
térmica se refere ao uso de calor para modificar a estrutura química da madeira, alterando-se assim outras propriedades.

Em ambos, o calor é utilizado para atingir seus objetivos e, por isso, podem ser comumente classificados como "térmicos". No entanto, um causa modificação química da estrutura da parede celular, enquanto o outro não tem esta capacidade. De fato, o objetivo da nota técnica do FPInnovations (2003) foi esclarecer que a modificação térmica é um processo distinto da secagem artificial para fins de controle fitossanitário e, portanto, não se podem utilizar ambas as nomenclaturas indiscriminadamente para um mesmo processo.

Dessa forma, percebe-se que o uso de tratamento térmico (ou tratamento com calor) não está tecnicamente errado do ponto de vista da Termodinâmica, mas pela generalidade, pode causar erros de interpretação. Além disso, já existe um processo mundialmente conhecido e consagrado, com definição prescrita em uma norma internacional e que recebe a designação tratamento térmico, que é a secagem artificial da madeira.

Para delineamentos experimentais em pesquisas científicas sobre o tema de modificação térmica, também é aceitável distinguir os métodos de estudo como tratamentos térmicos em oposição ao tratamento controle ou testemunha. Contudo, conforme exposto, a mesma nomenclatura não deve ser dada ao se mencionar o processo.

\section{Retificação térmica e termorretificação}

Ao se comparar as nomenclaturas técnicas das literaturas internacional e brasileira, identificou-se uma utilizada exclusivamente no Brasil, sem correspondente em Língua Inglesa: termorretificação. Esta palavra é uma aglutinação de retificação térmica, outra denominação do processo utilizada apenas no Brasil.

Conforme pesquisa bibliográfica, foi em um resumo apresentado no $7^{\circ}$ Congresso Florestal Brasileiro a primeira vez que retificação térmica constou em um trabalho científico publicado no Brasil (BRITO, 1993). De fato, salvo engano, este é o primeiro trabalho publicado no Brasil no tema. Posteriormente, foram publicados os primeiros artigos científicos com essa nomenclatura (PINCELLI; BRITO; CORRENTE, 2002; QUIRINO; VALE, 2002).

A palavra termorretificação foi apresentada no Brasil pela primeira vez em uma dissertação de mestrado (PINCELLI, 1999), em que o termo foi utilizado como sinônimo de retificação térmica. No trabalho de Pincelli, Brito e Corrente (2002) foi a primeira vez em que a utilização de ambos os termos como sinônimo foi publicada em uma revista científica. Desde então as pesquisas brasileiras no tema têm crescido e ambas nomenclaturas têm sido comumente empregadas, juntamente com as anteriormente mencionadas (tratamento térmico e modificação térmica).

Em busca realizada online no vocabulário ortográfico da Academia Brasileira de Letras em 30 de janeiro de 2019, no sítio eletrônico http://www.academia.org.br/nossa-lingua/busca-no-vocabulario, não foi encontrada a palavra termorretificação. Na Língua Portuguesa, o verbo retificar (ANJOS; FERREIRA, 2000) significa tornar reto, corrigir, emendar, dentre outros que não se aplicam à madeira. Assim, a retificação térmica, ou a sua forma aglutinada termorretificação, significam tornar a madeira reta, corrigida ou emendada com o uso de calor, o que não representa com exatidão as relações de causa e efeito que o processo causa na madeira. Isso viola um princípio fundamental de um conceito, conforme explicado por Ferrari (1982).

Provavelmente, os pesquisadores brasileiros cunharam as nomenclaturas retificação térmica $\mathrm{e}$ termorretificação por influência causada pelo contato com a literatura francesa do processo Retification, mencionado anteriormente. No entanto, Gérardin (2016) afirmou que o nome Retification dado ao processo é derivado da contração das palavras francesas réticulation e torréfaction, ou seja, trata-se de um nome fantasia criado para uma patente e que não possui equivalente em outras línguas. Por isso que os artigos publicados em Língua Inglesa não possuem termos equivalentes para retificação térmica e termorretificação. 


\section{Considerações Finais}

Para a padronização do processo e da madeira processada/produto originado, recomendam-se o uso das nomenclaturas técnicas modificação térmica e modificada termicamente, respectivamente.

Tecnicamente, não está errado o uso dos termos tratamento térmico e tratada termicamente e seus equivalentes tratamento com calor e tratada com calor (apesar destas serem formas mais raras). Contudo, para não incorrer em erros de interpretação, essas nomenclaturas devem ser evitadas porque são genéricas e tradicionalmente empregadas para o consagrado processo de secagem artificial da madeira.

Não se estimula o uso das nomenclaturas retificação térmica e termorretificação, bem como seus derivados retificada termicamente e termorretificada.

\section{Referências}

ALTGEN, M.; MILITZ, H. Photodegradation of thermally-modified Scots pine and Norway spruce investigated on thin micro-veneers. European Journal of Wood and Wood Products, Berlin, v. 74, 2015. DOI 10.1007/s00107-015-0980-3.

ANJOS, M.; FERREIRA, M. B. (Coord.). Miniaurélio século XXI escolar: o minidicionário da Língua Portuguesa. 4. ed. Rio de Janeiro: Nova Fronteira, 2000.

BAL, B. C. Physical properties of beech wood thermally modified in hot oil and in hot air at various temperatures. Maderas: Ciencia y Tecnología, Concepción, v. 17, n. 4, p. 789-798, 2015.

BASTANI, A.; ADAMOPOULOS, S.; MILITZ H. Gross adhesive penetration in furfurylated, N-methylol melamine-modified and heat-treated wood examined by fluorescence microscopy. European Journal of Wood and Wood Products, Berlin, 73, p. 635-642, $2015 \mathrm{a}$.

BASTANI, A.; ADAMOPOULOS, S.; MILITZ, H. Water uptake and wetting behaviour of furfurylated, N-methylol melamine modified and heat-treated wood. European Journal of Wood and Wood Products, Berlin, 73, p. 627-634, 2015b.

BATISTA, D. C. Modificação térmica da madeira de Eucalyptus grandis em escala industrial pelo processo brasileiro VAP HolzSysteme ${ }^{\circledR}$. 2012. 339 f. Tese (Doutorado em Engenharia Florestal) - Universidade Federal do Paraná, Curitiba, 2012.

BATISTA, D. C. et al. Microstructural aspects of thermally modified Eucalyptus grandis wood. Maderas: Ciencia y Tecnología, Concepción, v. 17, n. 3, p. 525-532, 2015.

BATISTA, D. C.; KLITZKE, R. J. Influência do tempo e temperatura de retificação térmica na umidade de equilíbrio da madeira de Eucalyptus grandis Hill ex Maiden. Scientia Forestalis, Piracicaba, v. 38, n. 86 , p. 255-261, 2010.

BATISTA, D. C.; TOMASELLI, I.; KLITZKE, R. J. Efeito do tempo e temperatura de modificação térmica na redução do inchamento máximo da madeira de Eucalyptus grandis Hill ex Maiden. Ciência Florestal, Santa Maria, v. 21, n. 3, p. 533-540, 2011.

BIZIKS, V. et al. One-stage thermo-hydro treatment (THT) of hardwoods: analysis of form stability after five soaking-drying cycles. Holzforschung, München, v. 69, n. 5, p. 563-571, 2015.

BORGES, L. M.; QUIRINO, W. F. Higroscopicidade da madeira de Pinus caribaea var. hondurensis tratado termicamente. Biomassa \& Energia, Dourados, v. 1, n. 2, p. 173-182, 2004.

BRITO, J. O. Estudo preliminar de retificação térmica da madeira de eucalipto. In: CONGRESSO FLORESTAL PANAMERICANO/ CONGRESSO FLORESTAL BRASILEIRO, 1/7., 1993, Curitiba. 
Anais... São Paulo: Sociedade Brasileira de Silvicultura, 1993. p. 774.

CALONEGO, F. W.; SEVERO, E. T. D.; FURTADO, E. L. Decay resistance of thermally-modified Eucalyptus grandis wood at $140^{\circ} \mathrm{C}, 160^{\circ} \mathrm{C}, 180^{\circ} \mathrm{C}, 200^{\circ} \mathrm{C}$ and $220^{\circ} \mathrm{C}$. Bioresource Technology, Amsterdam, v. 101, n. 23, p. 9391-9394, 2010.

CANDELIER, K. et al. Utilization of temperature kinetics as a method to predict treatment intensity and corresponding treated wood quality: durability and mechanical properties of thermally modified wood. Maderas: Ciencia y Tecnología, Concepción, v. 17, n. 2, p. 253-262, 2015.

CARvalho, A. G. et al. Colagem da madeira de Pinus termoretificada. Ciência da Madeira, Pelotas, v. 6, n. 3, p. 217-222, 2015.

ČERMÁK, P. et al. Analysis of dimensional stability of thermally modified wood affected by re-wetting cycles. BioResources, Raleigh, v. 10, n. 2, p. 3242-3253, 2015.

ČERMÁK, P. et al. The effect of wetting cycles on moisture behaviour of thermally modified Scots pine (Pinus sylvestris L.) wood. Journal of Materials Science, Berlin, v. 51, p. 1504-1511, 2016.

CIRULE, D. et al. Spectral sensitivity of thermally modified and unmodified wood. BioResources, Raleigh, v. 11, n. 1, p. 324-335, 2016.

ELAIEB, M. et al. Heat treatment of Tunisian soft wood species: effect on the durability, chemical modifications and mechanical properties. Maderas: Ciencia y Tecnología, Concepción, v. 17, n. 4, p. 699-710, 2015.

ESTEVES, B. M.; PEREIRA, H. M. Wood modification by heat treatment: a review. BioResources, Raleigh, v. 1, n. 4, p. 370-404, 2009.

EUROPEAN CONFERENCE ON WOOD MODIFICATION, 9., 2018, Arnhem. Programme... Disponível em: https://ecwm9.shr.nl/. Acesso em: 15 aug. 2018.

FABIYI, J. S.; OGUNLEYE, B. M. Mid-infrared spectroscopy and dynamic mechanical analysis of heat-treated obeche (Triplochiton scleroxylon) wood. Maderas: Ciencia y Tecnología, Concepción, v. 17, n. 1, p. 5-16, 2015.

FAJDIGA, G. et al. Compression test of thermally-treated beech wood: experimental and numerical analysis. BioResources, Raleigh, v. 11, n. 1, p. 223-234, 2016.

FERRARI, A. T. Metodologia da pesquisa científica. 1. ed. São Paulo: McGraw-Hill, 1982.

FIRMOLIN. Firmolin technologies. 2018. Disponível em: http://www.firmolin.com/index.php/en/. Acesso em: 15 aug. 2018.

FONTOURA, M. R. et al. Propriedades mecânicas e químicas da madeira de Hovenia dulcis Thunberg. tratada termicamente. Ciência da Madeira, Pelotas, v. 6, n. 3, p. 166-175, 2015.

FOODAND AGRICULTUREORGANIZATION OF THE UNITED NATIONS. NIMF-15: directrices para reglamentar el embalaje de madera utilizado en el comercio internacional. Roma: FAO, 2003.

FPINNOVATIONS. Thermally modified wood. 2003. Disponível em: http://www.valuetowood.ca/ imports/pdf/en/tech_profiles/TP-03-01E_TLihra_English.pdf. Acesso em: 15 ago. 2018.

GAFF, M. et al. Effect of selected parameters on the surface waviness in plane milling of thermally modified birch wood. BioResources, Raleigh, v. 10, n. 4, p. 7618-7626, 2015.

GAŠPARÍK, M. et al. Impact of thermal modification of spruce wood on screw direct withdrawal load resistance. BioResources, Raleigh, v. 10, n. 1, p. 1790-1802, 2015. 
GÉRARDIN, P. New alternatives for wood preservation based on thermal and chemical modification of wood - a review. Annals of Forest Science, Paris, v. 73, n. 3, p. 559-570, 2016. DOI 10.1007/s13595015-0531-4.

GUYONNET, R. Traitements termiques du bois Retification ${ }^{\circledR}$. 2007. Disponível em: https://www.emse. fr/fr/transfert/spin/depscientifiques/PC2M/retification/principes.html\#haut. Acesso em: 15 jan. 2016.

HAMADA, J. et al. Variations in the natural density of European oak wood affect thermal degradation during thermal modification. Annals of Forest Science, Paris, v. 73, n. 2, p. 277-286, 2016. DOI 10.1007/ s13595-015-0499-0.

HERMOSO, E. et al. Caracterización de la madera serrada de Pinus radiata modificada térmicamente. Maderas: Ciencia y Tecnología, Concepción, v. 17, n. 3, p. 493-504, 2015.

HILL, C. A. S. Wood modification: chemical, thermal and other processes. 1. ed. West Sussex: John Wiley \& Sons, 2006.

HOMAN, W. J.; JORISSEN, A. J. M. Wood modification developments. Heron, Delft, v. 49, n. 4, 2004.

HUGHES, M.; HILL, C.; PFRIEM, A. The toughness of hygrothermally modified wood. Holzforschung, München, v. 69, n. 7, p. 851-862, 2015.

HUGHES, M.; RAUTKARI, L.; JONES, D. [Website]. In: EUROPEAN CONFERENCE ON WOOD MODIFICATION, 8., 2015, Finland. Abstracts... Disponível em: http://ecwm8.aalto.fi/Extended\%20 abstract\%20Flyer-ECWM8.pdf. Acesso em: 15 jan. 2016.

INTERNATIONAL THERMOWOOD ASSOCIATION. International ThermoWood Association. 2018. Disponível em: http://www.thermowood.fi/. Acesso em: 15 ago. 2018.

INTERNATIONAL THERMOWOOD ASSOCIATION. ThermoWood ${ }^{\circledR}$ handbook. 2003. Disponível em: https://asiakas.kotisivukone.com/files/en.thermowood.kotisivukone.com/tiedostot/tw handbook_080813.pdf. Acesso em: 15 ago. 2018.

IWT MOLDRUP. Hydrothermal treatment of wood: efficient, fast and green. 2018. Disponível em: http://www.moldrupsystem.com/. Acesso em: 15 ago. 2018.

JAVED, M. A. et al. Magnetic resonance imaging study of water absorption in thermally modified pine wood. Holzforschung, München, v. 69, n. 7, p. 899-907, 2015.

KIM, J. S. et al. Chemical and ultrastructural changes of ash wood thermally modified using the thermo-vacuum process: Histo/cytochemical studies on changes in the structure and lignin chemistry. Holzforschung, München, v. 69, n. 5, p. 603-613, 2015a.

KIM, J. S. et al. Chemical and ultrastructural changes of ash wood thermally modified (TMW) using the thermo-vacuum process: II. Immuno cytochemical study of the distribution of noncellulosic polysaccharides.

Holzforschung, München, v. 69, n. 5, p. 615-625, 2015 b.

KORKUT, S.; AYTIN, A. Evaluation of physical and mechanical properties of wild cherry wood heattreated using the thermowood process. Maderas: Ciencia y Tecnología, Concepción, v. 17, n. 1, p. 171$178,2015$.

KUZMAN, M. K. et al. Effect of heat treatment on mechanical properties of selected wood joints. European Journal of Wood and Wood Products, Berlin, v. 73, p. 689-691, 2015.

KVIETKOVÁ, M. The effect of thermal treatment of birch wood on the cutting power of plain milling. BioResources, Raleigh, v. 10, n. 4, p. 8528-8538, 2015.

KVIETKOVÁ, M. et al. Surface quality of milled birch wood after thermal treatment at various 
temperatures. BioResources, Raleigh, v. 10, n. 4, p. 6512-6521, 2015.

KVIETKOVÁ, M.; GAŠPARÍK, M.; GAFF, M. Effect of thermal treatment on surface quality of beech wood after plane Milling. BioResources, Raleigh, v. 10, n. 3, p. 4226-4238, 2015.

LI, Y. et al. Quasi-static and dynamic nanoindentation to determine the influence of thermal treatment on the mechanical properties of bamboo cell walls. Holzforschung, München, v. 69, n. 7, p. 909-914, 2015.

LING, Z. et al. Microstructural and topochemical characterization of thermally modified poplar (Populus cathayaha) cell wall. BioResources, Raleigh, v. 11, n. 1, p. 786-799, 2016.

MILITZ, H.; ALTGEN, M. Processes and properties of thermally modified wood manufactured in Europe. In: SCHULTZ, T. P.; GOODELL, B.; NICHOLAS, D. D. (Ed.). Deterioration and protection of sustainable materials. Washington: Oxford University Press, 2014. p. 269-285.

OLÉOBOÍS. Oléoboís industries. 2018. Disponível em: http://www.oleobois-industries.com/en/?Home. Acesso em: 15 ago. 2018.

PALERMO, G. P. M. et al. Avaliação da superfície da madeira de Eucalyptus grandis Hill ex Maiden tratada termicamente. Ciência Florestal, Santa Maria, v. 25, n. 1, p. 145-152, 2015.

PANDEY, K. K.; KUMAR, S. V.; SRINIVAS, K. Inhibition of leaching of water soluble extractives of Pterocarpus marsupium by heat treatment. European Journal of Wood and Wood Products, Berlin, v. 74, 2015. DOI 10.1007/s00107-015-0964-.

PEARSON, H.; ORMARSSON, S.; GABBITAS, B. Nonlinear tensile creep behavior of radiata pine at elevated temperatures and different moisture contents. Holzforschung, München, v. 69, n. 7, p. 915-923, 2015 .

PIMENTEL-GOMES, F.; GARCIA, C. H. Estatística aplicada a experimentos agronômicos e florestais: exposição com exemplos e orientações para uso de aplicativos. Piracicaba: FEALQ, 2002.

PINCELLI, A. L. P. S. M. Efeito da termorretificação no envernizamento, colagem e cor da madeira de Eucalyptus saligna e Pinus caribaea var. hondurensis. 1999. 115 f. Dissertação (Mestre em Ciências) - Escola Superior de Agricultura Luiz de Queiroz, Universidade de São Paulo, Piracicaba, 1999.

PINCELLI, A. L. P. S. M.; BRITO, J. O.; CORRENTE, J. E. Avaliação da termorretificação sobre a colagem na madeira de Eucalyptus saligna e Pinus caribaea var. hondurensis. Scientia Forestalis, Piracicaba, v. 61, p. 122-132, jun. 2002.

PLATOWOOD. Platowood ${ }^{\circledR}$ makes the world a better place. 2018a. Disponível em: http://www. platowood.com/. Acesso em: 15 ago. 2018.

PLATOWOOD. The Platowood ${ }^{\circledR}$ process. 2018b. Disponível em: http://www.platowood.com/wpcontent/uploads/2015/08/The-Platowood-Process.pdf. Acesso em: 15 ago. 2018.

POUBEL, D. S. et al. Análises físicas e colorimétricas da madeira de Pinus sp. modificada termicamente. Scientia Forestalis, Piracicaba, v. 43, n. 107, p. 511-521, 2015.

QUIRINO, W. F.; VALE, A. T. Retificação térmica de Eucalyptus grandis. Floresta, Curitiba, nesp, p. 60-66, 2002.

RAPP, O. A. (Org.). Review on heat treatments of wood. In: SPECIAL SEMINAR COST ACTION E22: ENVIRONMENTAL OPTIMIZATION OF WOOD PROTECTION, Antibes, 2001. Proceedings... Hamburg: The Federal Research Centre for Forestry and Forest Products Information and Documentation, 2001. p. 1-68.

RINGMAN, R. et al. Effects of thermal modification on Postia placenta wood degradation dynamics: 
measurements of mass loss, structural integrity and gene expression. Wood Science and Technology, Berlin, 2015. DOI 10.1007/s00226-015-0791-z.

SAEI, A. M.; MOHEBBY, B.; ABDEH, M. R. Effects of oleothermal treatment and polydimethylsiloxane (PDMS) coating on natural weathering of beech and fir woods. Maderas: Ciencia y Tecnología, Concepción, v. 17, n. 4, p. 905-918, 2015.

SALMAN, S. et al. Decay and termite resistance of pine blocks impregnated with different additives and subjected to heat treatment. European Journal of Wood and Wood Products, Berlin, v. 74, n. 1, p. 37 42, 2016.

SANDBERG, D.; KUTNAR, A. Thermally modified timber: recent developments in Europe and North America. Wood and Fiber Science, Monona, v. 48, nesp, p. 28-39, 2016.

SIMPSON, W. T. (Ed.). Dry kiln operator's manual. Madison: United States Department of Agriculture; Forest Service; Forest Products Laboratory, 1991.

SIVRIKAYA, H. et al. Comparative biological resistance of differently thermal modified wood species against decay fungi, Reticulitermes grassei and Hylotrupes bajulus. Maderas: Ciencia y Tecnología, Concepción, v. 17, n. 3, p. 559-570, 2015.

SMITH, J. M.; VAN, N. H. C.; ABBOTT, M. M. Introdução à termodinâmica da engenharia química. Rio de Janeiro: LTC, 2011.

SONDEREGGER, W. et al. On-line monitoring of hygroscopicity and dimensional changes of wood during thermal modification by means of neutron imaging methods. Holzforschung, München, v. 69, n. 1, p. 87-95, 2015.

TAKESHITA, S.; JANKOWSKY, I. P. Redução na movimentação dimensional da madeira de Jatobá (Hymenaea sp.) e Muiracatiara (Astronium sp.) submetidas a tratamento térmico adicional. Scientia Forestalis, Piracicaba, v. 43, n. 106, p. 345-352, 2015.

TIMURA. Thermo wood: patented technology from Timura. 2018. Disponível em: http:// https://www. timurawood.com/wood-manufacturer/thermo-wood. Acesso em: 15 ago. 2018.

TJEERDSMA, B. et al. Characterization of thermally modified wood: molecular reasons for wood performance improvement. Holz als Roh-und Werkstoff, Berlin, v. 56, p. 149-153, 1998.

TODOROVIĆ, N.; POPOVIĆ, Z.; MILIĆ, G. Estimation of quality of thermally modified beech wood with red heartwood by FT-NIR spectroscopy. Wood Science and Technology, Berlin, v. 49, p. 527-549, 2015.

TORNIAINEN, P.; ELUSTONDO, D.; DAGBRO, O. Industrial validation of the relationship between color parameters in thermally modified spruce and pine. BioResources, Raleigh, v. 11, n. 1, p. 1369-1381, 2016.

TRCALA, M.; ČERMÁK, P. Numerical analysis of temperature profiles during thermal modification of wood: chemical reactions and experimental verification. Holzforschung, München, v. 69, n. 3, p. 321$328,2015$.

TUKIAINEN, P.; HUGHES, M. The effect of elevated temperature and high moisture content on the fracture behaviour of thermally modified spruce. Journal of Materials Science, Berlin, v. 51, n. 3, p. 1437-1444, 2016.

WANG, W. et al. Thermal modification of southern pine combined with wax emulsion preimpregnation: effect on hydrophobicity and dimensional stability. Holzforschung, München, v. 69, n. 4, p. 405-413, 2015. 
WDE MASPELL. High temperature treatment. 2018. Disponível em: http:/www.wde-maspell.com/ high-temperature-treatment.html. Acesso em: 15 ago. 2018.

WILLEMS, W. et al. Quality control methods for thermally modified wood. Holzforschung, München, v. 69 , n. 7, p. 875-884, 2015.

WOOD TREATMENT TECHNOLOGY. Thermo treatment plant - producing thermally modified timber: TMT wood. 2018. Disponível em: http:// http://wtt.global/products/thermo-treatment/. Acesso em: 15 ago. 2018.

XIE; LIU, Y.; SUN, Y. Heat treated wood and its development. Journal of Forestry Research, Berlin, v. 13, n. 2, p. 224-230, 2002.

YALCIN, M.; SAHIN, H. I. Changes in the chemical structure and decay resistance of heat-treated narrowleaved ash wood. Maderas: Ciencia y Tecnología, Concepción, v. 17, n. 2, p. 435-446, 2015.

YANG, H.; CHENG, W.; HAN, G. Wood modification at high temperature and pressurized steam: a relational model of mechanical properties based on a neural network. BioResources, Raleigh, v. 10, n. 3, p. 5758-5776, 2015.

YANG, Y. et al. Influences of thermo-vacuum treatment on colors and chemical compositions of alder birch wood. BioResources, Raleigh, v. 10, n. 4, p. 7936-7945, 2015.

ZANUNCIO, A. J. V. et al. Effect of extractives on wood color of heat treated Pinus radiata and Eucalyptus pellita. Maderas: Ciencia y Tecnología, Concepción, v. 17, n. 4, p. 857-864, 2015.

ZAUER, M. et al. Thermal modification of European beech at relatively mild temperatures for the use in electric bass guitars. European Journal of Wood and Wood Products, Berlin, v. 74, p. 43-48, 2016.

ZHAN, J.; AVRAMIDIS, S. Needle fir wood modified by surface densification and thermal post-treatment: hygroscopicity and swelling behavior. European Journal of Wood and Wood Products, Berlin, v. 74, p. 49-56, 2016.

ZHANG, T. et al. Effects of heat treatment on physical-mechanical properties of Eucalyptus regnans. BioResources, Raleigh, v. 10, n. 2, p. 3531-3540, 2015.

ZIGON, J. et al. The influence of heat and chemical treatments of beech wood on the shear strength of welded and UF bonded specimens. European Journal of Wood and Wood Products, Berlin, v. 73, p. 685-687, 2015. 